\title{
Cost-effectiveness of artemisinin- naphthoquine versus artemether-lumefantrine for the treatment of uncomplicated malaria in Papua New Guinean children
}

Brioni R. Moore ${ }^{1,2 \dagger}$, Wendy A. Davis ${ }^{2 \dagger}$, Philip M. Clarke ${ }^{3}$, Leanne J. Robinson ${ }^{4,5,6}$, Moses Laman $^{4 \ddagger}$ and Timothy M. E. Davis ${ }^{2 *} \neq 0$

\begin{abstract}
Background: A recent randomized trial showed that artemisinin-naphthoquine (AN) was non-inferior to artemether-lumefantrine (AL) for falciparum malaria and superior for vivax malaria in young Papua New Guinean children. The aim of this study was to compare the cost-effectiveness of these two regimens.

Methods: An incremental cost-effectiveness analysis was performed using data from 231 children with Plasmodium falciparum and/or Plasmodium vivax infections in an open-label, randomized, parallel-group trial. Recruited children were randomized 1:1 to receive once daily AN for 3 days with water or twice daily AL for 3 days given with fat. World Health Organisation (WHO) definitions were used to determine clinical/parasitological outcomes. The cost of transport between the home and clinic, plus direct health-care costs, served as a basis for determining each regimen's incremental cost per incremental treatment success relative to AL by Day 42 and its cost per life year saved.

Results: In the usual care setting, AN was more effective for the treatment of uncomplicated malaria in children aged 0.5-5.9 years. AL and AN were equally efficacious for the treatment of falciparum malaria, however AN had increased anti-malarial treatment costs per patient of $\$ 10.46$, compared with $\mathrm{AL}$. AN was the most effective regimen for treatment of vivax malaria, but had increased treatment costs of $\$ 14.83$ per treatment success compared with AL.

Conclusions: Whilst AN has superior overall efficacy for the treatment of uncomplicated malaria in PNG children, AL was the less costly regimen. An indicative extrapolation estimated the cost per life year saved by using AN instead of AL to treat uncomplicated malaria to be $\$ 12,165$ for girls and $\$ 12,469$ for boys (discounted), which means AN may not be cost-effective and affordable for PNG at current cost. However, AN may become acceptable should it become WHO prequalified and/or should donated/subsidized drug supply become available.
\end{abstract}

Keywords: Uncomplicated malaria, Artemether-lumefantrine, Artemisinin-naphthoquine, Children, Cost-effectiveness

\footnotetext{
*Correspondence: tim.davis@uwa.edu.au

†Brioni R. Moore and Wendy A. Davis contributed equally as first author

${ }^{\ddagger}$ Moses Laman and Timothy M. E. Davis contributed equally as senior

author

${ }^{2}$ School of Medicine and Pharmacology, University of Western Australia,

Perth, WA, Australia

Full list of author information is available at the end of the article
} 


\section{Background}

Although malaria remains a major global health issue with nearly half the world's population still at risk and ongoing transmission in 91 countries, substantial progress has been made towards reducing the burden of the disease [1]. The incidence of malaria declined by $21 \%$ between 2000 and 2015 [1], and the proportion of global disability adjusted life years attributable to malaria fell from 3.5 to $2.3 \%$ over same period representing a drop in ranking relative to other causes from 7 th to 14th [2]. This improvement reflects more effective vector control and case management [1].

Artemisinin-based combination therapy (ACT) is the World Health Organization (WHO) recommended firstline treatment for uncomplicated malaria [1]. Five different artemisinin-based combinations, artemether-lumefantrine (AL), artesunate-amodiaquine, artesunate-mefloquine, dihydroartemisinin-piperaquine (DHA-PQP) and artesunate-sulfadoxine-pyrimethamine, currently meet the stringent WHO regulatory standards for prequalification with the choice of ACT based, at least in part, on local parasite drug resistance [1]. The incorporation of ACTs into malaria control programmes has contributed to the global decline in malaria-related mortality and morbidity, but effective therapy remains a challenge in geo-epidemiological settings where there is transmission of multiple Plasmodium species [3]. Papua New Guinea (PNG) is a case in point. In 2011, PNG national malaria treatment guidelines were modified to include $\mathrm{AL}$ as firstline treatment for uncomplicated Plasmodium falciparum and Plasmodium vivax infections [4]. These changes reflected WHO contemporary recommendations and were underpinned by the results of a randomized clinical trial that demonstrated that $\mathrm{AL}$ was the most efficacious [5] and cost-effective [6] ACT for falciparum malaria in PNG. Although DHA-PQP was more efficacious in vivax malaria, the cost and complexity of having different firstline treatments for cases of falciparum and vivax malaria, meant that it was included as an alternative second-line regimen $[4,5]$. Given that potentially preventable morbidity and mortality due to vivax malaria despite AL therapy remained a concern [7], a search for more broadly effective and affordable formulations of ACT was recommended [5].

Of the few potential alternative ACT, artemisininnaphthoquine (AN) was one candidate. This ACT was already available in the private sector in PNG as singledose treatment for uncomplicated malaria despite not being prequalified. Preliminary pharmacokinetic and safety studies in PNG children provided good evidence that the recommended single AN dose given daily for 3 days (a duration recommended by the WHO for all $\mathrm{ACT}$ medicines to increase cure rates and reduce the development of parasite resistance [8]) would be safe, well tolerated and efficacious treatment for uncomplicated malaria $[9,10]$, with the long elimination half-life of naphthoquine $\left(t_{1 / 2}=23\right.$ days) [10] likely to provide more extended suppression of late post-treatment $P$. vivax emergence than the piperaquine component of DHA-PQP.

A randomized comparative safety and efficacy trial of $\mathrm{AL}$ and $\mathrm{AN}$ was, therefore, performed in young PNG children with uncomplicated $P$. falciparum and $P$. vivax infections in coastal Madang Province [11]. The results showed that AN was non-inferior to AL for falciparum malaria with the same high cure rate, but that it was superior to AL for vivax malaria. However, the cost of using three times the recommended dose of a non-subsidized ACT is likely to be relatively high $[12,13]$, which would be an impediment to its uptake in developing countries with similar epidemiology to that in malariaendemic areas of PNG. The aim of the present study was, therefore, to assess the relative cost-effectiveness of $\mathrm{AL}$ and $\mathrm{AN}$ for the treatment of uncomplicated malaria in Melanesian children aged $0.5-5.9$ years.

\section{Methods \\ Patients}

Between 28 March 2011 and 22 April 2013, an openlabel, randomized, parallel-group trial of AL and AN was conducted at the Mugil and Alexishafen Health Centres (Australian New Zealand Clinical Trials Registry ACTRN12610000913077). The trial was approved by the PNG Institute of Medical Research Review Board, the Medical Research Advisory Committee of PNG, and the University of Western Australia Human Research Ethics Committee. Children aged 0.5-5.9 years presenting with blood slide positive uncomplicated falciparum or vivax malaria were eligible to participate. Full details of study procedures have been published [11]. Using a computergenerated block randomization (24 children per site), participants were allocated $1: 1$ to either AL $(1.7 \mathrm{mg} /$ $\mathrm{kg}$ artemether plus $10 \mathrm{mg} / \mathrm{kg}$ lumefantrine; Novartis Pharma, Basel, Switzerland) twice daily for 3 days, or to AN (20 mg/kg artemisinin plus $8 \mathrm{mg} / \mathrm{kg}$ naphthoquine; Kunming Pharmaceutical Corporation (KPC), Yunnan, China) daily for 3 days. As recommended by the manufacturer, AL was administered as 1-3 whole tablets per dose with $250 \mathrm{ml}$ of milk, whilst AN was given as 1-4 whole tablets with water, with the number of tablets given per dose based on manufacturer recommended weight/dose ranges. Children who vomited within $30 \mathrm{~min}$ of drug administration were retreated. The present economic evaluation included all children randomized and followed according to the study protocol (198 with falciparum malaria, 47 with vivax malaria; see Fig. 1). 


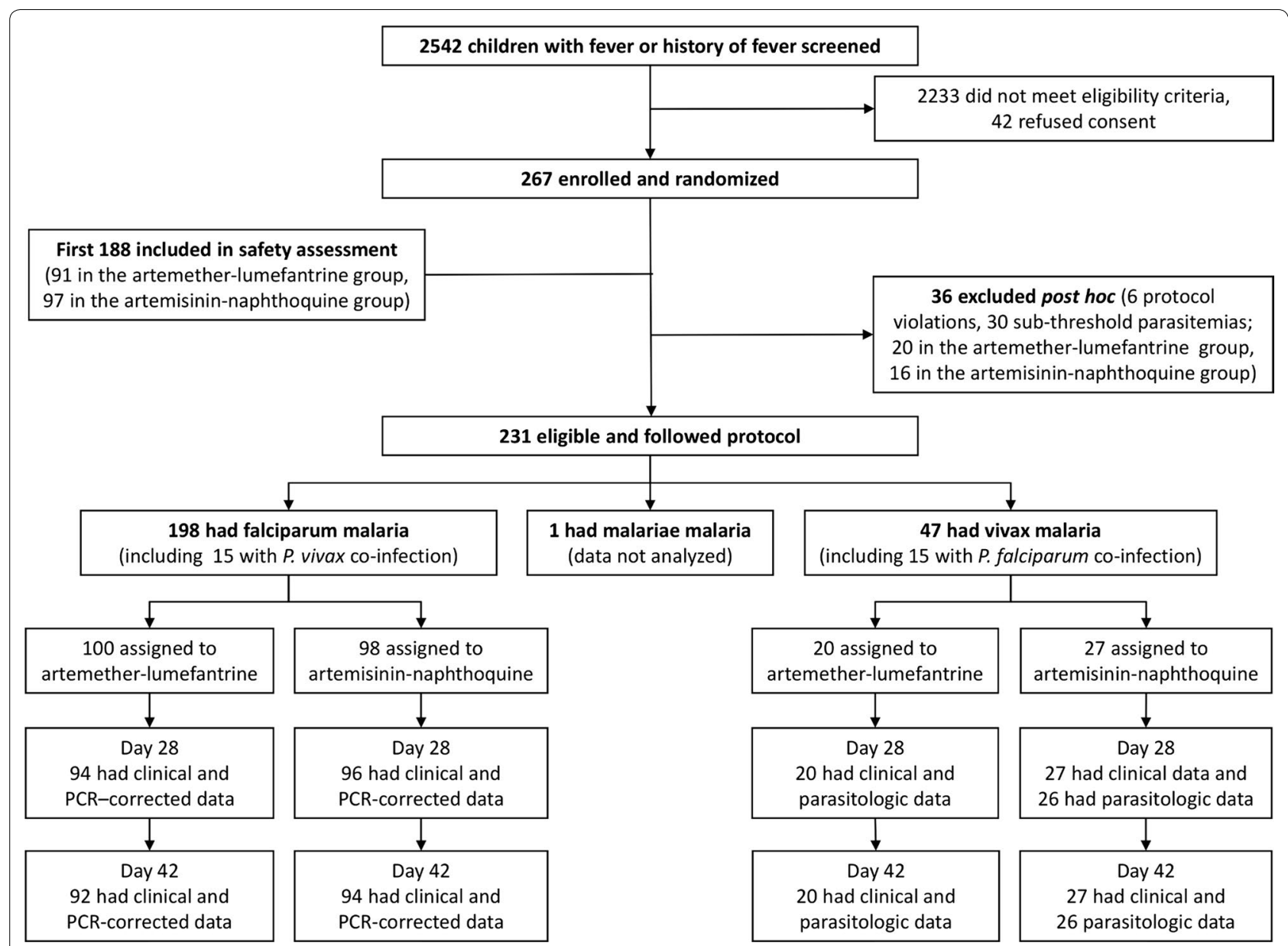

Fig. 1 Consort diagram showing numbers of patients from screening to Day 42 assessment. PCR-corrected denotes correction for re-infections identified by PCR genotyping of polymorphic parasite loci (adapted from [1 1])

\section{Clinical and parasitological methods}

An initial standardized clinical assessment including measurement of the axillary temperature was performed and blood was drawn for blood film microscopy, measurement of haemoglobin $(\mathrm{Hb})$ and blood glucose, hepatic and renal function, and a full blood count [11]. A modified assessment was repeated on Days 1, 2, 3, 7, 14, 28 and 42 . All blood films were examined by two independent microscopists with parasite density calculated from the number of parasites per 200-500 leucocytes and an assumed leucocyte count of $8000 / \mu \mathrm{l}$.

Efficacy was assessed using WHO definitions [14], specifically (i) early treatment failure (ETF) or the development of signs of severity or an inadequate parasitological response by Day 3, (ii) late parasitological failure (LPF) or the development of parasitaemia between Days 4 and 42, (iii) late clinical failure (LCF) if the LPF was accompanied by fever, or (iv) adequate parasitological and clinical response (ACPR) otherwise. These outcomes were corrected for re-infection by polymerase chain reaction
(PCR) genotyping of the falciparum malaria cases, while $P$. vivax recrudescence was determined by genotyping [11]. No children developed severe malaria during follow-up. Two LCFs were identified at the Day 42 assessment and received $\mathrm{AL}$ treatment as per protocol and PNG national treatment guidelines.

\section{Economic analyses}

The perspective of the present analyses was societal. Direct health care costs (AL and AN treatment costs, visits to health centres and tests, and a course of rescue antimalarial therapy when required) plus travel costs were estimated. An incremental cost-effectiveness analysis was performed in which the net costs and net effectiveness of AN were compared with those of conventional AL treatment and expressed as ratios. All analyses and comparisons were performed on both a per protocol (PP) and modified intention to treat (mITT) basis. The PP analyses included children with complete follow-up or confirmed treatment failure, and excluded those treated for malaria 
without confirmatory microscopy or who defaulted from follow-up. These excluded patients were retained in the mITT which utilized i) a worst-case approach (ETF assumed for Day 3 exclusions, LPF/LCF otherwise) and ii) a best-case approach (all missing follow-up blood films assumed parasite-negative). In a secondary analysis, we extrapolated outcomes to estimate the increase in life expectancy of the most effective treatment based on estimated mortality associated with $P$. falciparum and remaining life expectancy.

For each patient, standardized data were collected at each scheduled clinic visit and at extra, unscheduled "sick day" visits. These included doses of all drugs used for treating malaria, its symptoms and its complications (trial medication, rescue $\mathrm{AL}$, paracetamol, iron and folate supplements). Unit costs were obtained from the PNG National Department of Health [15], Interpath Services Pty Ltd (Heidelberg West, Victoria, Australia), HemoCue Australia Pty Ltd (Tumbi Umbi, New South Wales, Australia), Access Bio Inc. (Somerset, NJ, USA), participating clinics and local suppliers (see Table 1), and were combined with resource volumes to obtain a net cost per patient during follow-up. Mean net costs and associated 95\% confidence intervals (CI) were calculated for each treatment arm. Costs are reported undiscounted due to the relative brevity of the trial, and in 2012 US\$ values using the average exchange rate during 2012 of 1 PNG Kina $(\mathrm{PGK})=$ US\$0.490757 [16].

All study participants were scheduled to attend eight times including Day 0 but excluding sick days. However, the frequency and type of visits are different in a usual care (non-trial) situation. Therefore, a complementary analysis, in which costs of AL or AN treatment are based on a single clinic visit at which malaria is diagnosed and treated, was conducted. The same number of subsequent sick day visits was assumed except for ETFs where the scheduled Day 1-3 clinic visit was replaced by a sick day visit. Each patient's actual trial visit costs were replaced by the estimated standard practice cost depending on allocation. It was assumed that no-cost forms of fat (from a normal diet or breastfeeding in the case of young infants) were consumed with AL.

The primary endpoint of the trial was treatment failure by Day 42 [5]. A secondary analysis of the lifetime benefits of using AN for P. falciparum was performed by extrapolating the benefits using lifetables to estimate the potential life years saved. All comparisons were carried out separately for $P$. falciparum and $P$. vivax on both a PP and a mITT basis. All results are reported as means and SDs or mean differences and $95 \%$ CIs. The CIs for the key incremental cost-effectiveness ratios (ICERs) were estimated using the bootstrap approach with 1000 repeated random samples drawn with replacement from the original data. Bootstrap confidence intervals were constructed with the bias-corrected percentile method [17]. The effect of assumptions on main results was examined by sensitivity analyses involving undertaking a costeffectiveness analysis using best and worst case mITT assumptions. Data were analysed using IBM SPSS Statistics 22 (IBM Corporation, Armonk, New York, USA).

\section{Results}

The 186 children in the PP P. falciparum analysis were of mean \pm SD age $3.7 \pm 1.3$ (range $0.8-5.9$ ) years and $53.8 \%$ were boys, while $41.3 \%$ of the 46 children in the PP $P$. vivax analysis were boys and the mean age was $3.1 \pm 1.2$ (range 0.5-5.8) years.

\section{Costs}

Table 2 shows the mean cost per patient and the mean cost difference between AL and AN over the duration of the study by category of cost and allocation for P. falciparum and $P$. vivax in both trial and usual care settings. For P. falciparum, AN had increased anti-malarial treatment costs for each patient of $\$ 10.46$ (95\% CI \$9.77-\$11.16) on average compared with AL. There were no significant differences between AL and AN in other costs. Total usual care costs were significantly higher in the AN group compared with the AL group.

For $P$. vivax infections, AN increased anti-malarial treatment costs for each patient by an average of $\$ 11.05$ (95\% CI \$9.93-\$12.16) compared with AL (see Table 2). There were no significant differences between $\mathrm{AL}$ and AN therapies in other costs. Total usual care treatment costs were significantly higher in the AN group compared with AL treatment.

\section{Outcomes}

In the PP P. falciparum analysis of the 186 children who completed the trial, 184 (98.9\%) had an ACPR with the highest rate in the AN group (100\%) compared with $97.8 \%$ in the AL group [11]. For the 32 children who attended on Day 42, the rate of ACPR for $P$. vivax was $100 \%$ in the AN group compared with $30.0 \%$ in the AL group [11]. Table 3 documents the proportion of treatment successes and the costs for each malaria species and each type of analysis (PP and mITT) for a usual care setting. The incremental number of successes and costs together with the ICER are also shown for AN compared with AL.

\section{Cost-effectiveness}

The primary measure of cost-effectiveness is the incremental cost per incremental treatment success relative to the comparator AL. In a usual care setting, AN was more effective but more costly than AL (see scatterplot 
Table 1 Main unit costs and sources for the two treatment arms

\begin{tabular}{|c|c|c|c|c|}
\hline Item & Unit cost (2012 PGK) & Unit cost (2012 US\$) & Source & Comments \\
\hline \multicolumn{5}{|l|}{ Health clinic visit } \\
\hline Outpatient & 3.00 & 1.472 & $\begin{array}{l}\text { Alexishafen/Mugil Health } \\
\text { Centres }\end{array}$ & $\begin{array}{l}\text { Includes examination by nurse } \\
\text { and treatment }\end{array}$ \\
\hline Inpatient & 10.00 & 4.908 & $\begin{array}{l}\text { Alexishafen/Mugil Health } \\
\text { Centres }\end{array}$ & All treatment costs \\
\hline \multicolumn{5}{|c|}{ Transport cost to Alexishafen Health Centre } \\
\hline PMV (return) & 4.00 & 1.963 & Alexishafen Health Centre & $\begin{array}{l}\text { For average distance (PGK2 } \\
\text { close-PGK6 far) }\end{array}$ \\
\hline $\begin{array}{l}\text { Hire vehicle (night transport } \\
\text { after PMV hours) }\end{array}$ & 35.00 & 17.176 & Alexishafen Health Centre & Range PGK20-PGK50 \\
\hline Ambulance (emergency) & 30.00 & 14.723 & Alexishafen Health Centre & \\
\hline \multicolumn{5}{|c|}{ Transport cost to Mugil Health Centre } \\
\hline PMV (return) & 6.00 & 2.945 & Mugil Health Centre & $\begin{array}{l}\text { For average distance (PGK2 } \\
\text { close-PGK10 far) }\end{array}$ \\
\hline $\begin{array}{l}\text { Hire vehicle (night transport } \\
\text { after PMV hours) }\end{array}$ & 40.00 & 19.630 & Mugil Health Centre & Range PGK30- PGK50 \\
\hline Ambulance (emergency) & 30.00 & 14.723 & Mugil Health Centre & \\
\hline \multicolumn{5}{|l|}{ Malaria treatment } \\
\hline$A+L$ & 0.396 & 0.194 & $\begin{array}{l}\text { PNG National Department of } \\
\text { Health, } 2012\end{array}$ & National standard treatment \\
\hline$A+N$ & 3.75 & 1.840 & $\begin{array}{l}\text { Local pharmacy over-the- } \\
\text { counter }\end{array}$ & \\
\hline \multicolumn{5}{|l|}{ Other medications } \\
\hline $\begin{array}{l}\text { Paracetamol syrup } \\
120 \mathrm{mg} / 5 \mathrm{ml}\end{array}$ & $0.010 / \mathrm{ml}$ & $0.005 / \mathrm{ml}$ & $\begin{array}{l}\text { PNG National Department of } \\
\text { Health, } 2012\end{array}$ & $\begin{array}{l}15 \mathrm{mg} / \mathrm{kg} \text { given if axillary tem- } \\
\text { perature }>37.5^{\circ} \mathrm{C}\end{array}$ \\
\hline Paracetamol tablets, $500 \mathrm{mg}$ & 0.011 & 0.005 & $\begin{array}{l}\text { PNG National Department of } \\
\text { Health, } 2012\end{array}$ & $\begin{array}{l}15 \mathrm{mg} / \mathrm{kg} \text { given if axillary tem- } \\
\text { perature }>37.5^{\circ} \mathrm{C}\end{array}$ \\
\hline $\begin{array}{l}\text { Paracetamol suppository, } \\
250 \mathrm{mg}\end{array}$ & 0.226 & 0.111 & $\begin{array}{l}\text { PNG National Department of } \\
\text { Health, } 2012\end{array}$ & $\begin{array}{l}15 \mathrm{mg} / \mathrm{kg} \text { given if axillary tem- } \\
\text { perature }>37.5^{\circ} \mathrm{C}\end{array}$ \\
\hline Albendazole $200 \mathrm{mg}$ tablet & 0.025 & 0.012 & $\begin{array}{l}\text { PNG National Department of } \\
\text { Health, } 2012\end{array}$ & $\begin{array}{l}\text { For hookworm on Day } 42 \text { if } \\
\qquad \mathrm{Hb}<90 \mathrm{~g} / \mathrm{l}\end{array}$ \\
\hline $\begin{array}{l}\text { Fefol (FeSO } 200 \text { mg, folic acid } \\
\quad 0.4 \mathrm{mg})\end{array}$ & 0.027 & 0.013 & $\begin{array}{l}\text { PNG National Department of } \\
\text { Health, } 2012\end{array}$ & Day 42 if $\mathrm{Hb}<90 \mathrm{~g} / \mathrm{l}$ \\
\hline Amodiaquine $100 \mathrm{mg}$ tablet & 0.024 & 0.012 & $\begin{array}{l}\text { PNG National Department of } \\
\text { Health, } 2012\end{array}$ & $\begin{array}{l}\text { Day } 42 \text { if } \mathrm{Hb}<90 \mathrm{~g} / \mathrm{l} \text { and spleen } \\
\text { grade } \geq 3\end{array}$ \\
\hline \multicolumn{5}{|l|}{ Tests } \\
\hline Rapid diagnostic test & 2.241 & 1.100 & Access Bio Inc. & $\begin{array}{l}\text { CareStart Malaria HRP2/pLDH (Pf/ } \\
\text { PAN) Combo; Baseline }\end{array}$ \\
\hline $\begin{array}{l}\text { HemoCue } \mathrm{Hb} \text { point of care } \\
\text { (POC) test }\end{array}$ & 3.454 & 1.695 & HemoCue Australia Pty Ltd & \\
\hline HemoCue glucose POC test & 3.896 & 1.912 & HemoCue Australia Pty Ltd & \\
\hline $\begin{array}{l}\text { Malaria microscopy, blood } \\
\text { slide and reading }\end{array}$ & 3.580 & 1.757 & Interpath, national salary & $\begin{array}{l}\text { Days } 0-42 \text {; includes price of } \\
\text { blood slide, stain, } 15 \text { min salary } \\
\text { to read slide }\end{array}$ \\
\hline \multicolumn{5}{|l|}{ Other } \\
\hline $\begin{array}{l}\text { Milk ( } 250 \mathrm{ml} \text { tetra packs full } \\
\text { cream) }\end{array}$ & 1.708 & 0.838 & $\begin{array}{l}\text { K41.00/24 packs wholesale } \\
\text { (2011) }\end{array}$ & \\
\hline $\begin{array}{l}\text { Syringe disposable } 10 \mathrm{ml} \text { with } \\
21-\mathrm{G} \text { needle }\end{array}$ & 0.210 & 0.103 & $\begin{array}{l}\text { PNG National Department of } \\
\text { Health, } 2012\end{array}$ & \\
\hline Gloves, disposable & 0.136 & 0.067 & $\begin{array}{l}\text { PNG National Department of } \\
\text { Health, } 2012\end{array}$ & PGK6.82/box non-sterile gloves \\
\hline Blood lancet, disposable & 0.032 & 0.016 & $\begin{array}{l}\text { PNG National Department of } \\
\text { Health, } 2012\end{array}$ & PGK6.38/box of 200 lancets \\
\hline $\begin{array}{l}\text { Alcohol wipe } \times 1 \text { each blood } \\
\text { sample }\end{array}$ & 0.017 & 0.008 & $\begin{array}{l}\text { PNG National Department of } \\
\text { Health, } 2012\end{array}$ & PGK1.74/box of 100 sterile wipes \\
\hline
\end{tabular}


Table 1 continued

\begin{tabular}{lllll}
\hline Item & Unit cost (2012 PGK) & Unit cost (2012 US\$) & Source & Comments \\
\hline $\begin{array}{l}\text { Cotton wool swab } \times 1 \text { each } \\
\text { blood sample }\end{array}$ & 0.007 & 0.003 & $\begin{array}{l}\text { PNG National Department of } \\
\text { Health, 2012 }\end{array}$ & PGK3.55/500 g pack \\
\hline
\end{tabular}

Per tablet or test except where indicated otherwise

Table 2 Per protocol analysis showing mean \pm SD per patient costs and mean $(95 \% \mathrm{Cl})$ cost differences in 2012 US\$ for AL vs. AN given for falciparum (upper panel) or vivax (lower panel) malaria in usual care and trial settings

\begin{tabular}{|c|c|c|c|}
\hline & $A L$ & AN & AN vs. AL \\
\hline \multicolumn{4}{|l|}{ Falciparum malaria } \\
\hline Number & 92 & 94 & \\
\hline $\begin{array}{c}\text { Anti-malarial } \\
\text { treatment }\end{array}$ & $1.58 \pm 0.56$ & $12.04 \pm 3.35$ & $\begin{array}{l}10.46(9.77 \text { to } \\
11.16)^{*}\end{array}$ \\
\hline Paracetamol & $0.003 \pm 0.002$ & $0.003 \pm 0.003$ & $\begin{array}{l}0.000(-0.001 \text { to } \\
0.001)\end{array}$ \\
\hline Clinic visits & $5.53 \pm 1.63$ & $5.23 \pm 0.77$ & $\begin{array}{l}-0.29(-0.66 \text { to } \\
0.08)\end{array}$ \\
\hline Total (usual care) & $7.66 \pm 2.29$ & $17.28 \pm 3.40$ & $9.61(8.78 \text { to } 10.45)^{*}$ \\
\hline $\begin{array}{l}\text { Excess costs for } \\
\text { trial }\end{array}$ & $54.71 \pm 1.31$ & $49.56 \pm 1.54$ & $\begin{array}{l}-5.15(-5.56 \text { to } \\
-4.74)^{*}\end{array}$ \\
\hline Total (trial) & $62.37 \pm 3.21$ & $66.84 \pm 3.83$ & $4.46(3.44 \text { to } 5.49)^{*}$ \\
\hline \multicolumn{4}{|l|}{ Vivax malaria } \\
\hline Number & 20 & 26 & \\
\hline $\begin{array}{l}\text { Anti-malarial } \\
\text { treatment }\end{array}$ & $1.41 \pm 0.48$ & $12.46 \pm 2.72$ & $\begin{array}{l}11.05(9.93 \text { to } \\
12.16)^{*}\end{array}$ \\
\hline Paracetamol & $0.003 \pm 0.003$ & $0.002 \pm 0.002$ & $\begin{array}{l}-0.000(-0.002 \text { to } \\
0.001)\end{array}$ \\
\hline Clinic visits & $5.12 \pm 0.00$ & $5.32 \pm 1.04$ & $0.20(-0.27$ to 0.67$)$ \\
\hline Total (usual care) & $7.41 \pm 1.16$ & $17.78 \pm 3.20$ & $\begin{array}{l}10.38(9.00 \text { to } \\
11.76)^{*}\end{array}$ \\
\hline $\begin{array}{l}\text { Excess costs for } \\
\text { trial }\end{array}$ & $54.72 \pm 0.13$ & $49.76 \pm 0.43$ & $\begin{array}{l}-4.96(-5.16 \text { to } \\
-4.76)^{*}\end{array}$ \\
\hline Total (trial) & $62.12 \pm 1.14$ & $67.54 \pm 3.45$ & $5.42(3.95 \text { to } 6.89)^{*}$ \\
\hline
\end{tabular}

${ }^{*} P<0.001$ vs. AL; negative cost differences indicate cost-savings associated with AN

of estimated joint density of incremental costs and incremental effects of AN vs. AL obtained by bootstrap resampling in Fig. 2). For P. falciparum, the average excess cost per treatment success for AN when compared with AL was $\$ 436.82$ with a $95 \%$ bootstrap bias-corrected confidence interval of $\$ 200.23-\$ 941.29$ and a $68.3 \%$ chance that the incremental cost-effectiveness ratio was below $\$ 500$ per treatment success. For $P$. vivax (see Fig. 2), AN was more effective than $\mathrm{AL}$, but more costly with an average excess cost per treatment success of $\$ 14.83$ in a usual care setting when compared with $\mathrm{AL}$ with a $95 \%$ bootstrap bias-corrected confidence interval of $\$ 11.06$ to $\$ 22.17$ and a very high probability $(99.7 \%)$ that the excess cost per success was $<\$ 25$.

\section{Cost per life year saved if $A L$ is replaced by AN}

A girl and boy aged between 1 and 4 years old in 2012 in PNG could expect to live another 66.6 and 62.1 years, respectively [18]. In PNG in 2012, there were 150,195 reported confirmed cases of malaria and 381 reported malaria deaths, a mortality rate of $0.25 \%$ [19], with both cases and deaths concentrated in younger age groups which have yet to develop immunity. The incremental success of AN over AL was $2.2 \%$ (100\% vs. 97.8\%) in children $0.5-5.9$ years old. The number of deaths from P. falciparum prevented per 1000 cases when treated with $\mathrm{AN}$ instead of AL is thus estimated to be $0.0025 \times 1000 \times 0.022=0.055$. The increase in average life expectancy for 1000 cases of $P$. falciparum treated with AN is approximately $0.055 \times 66.6$ years for girls $=3.66$ years (or 1.64 years when discounted at $3 \%$ ) and $0.055 \times 62.1$ years for boys $=3.42$ years (or 1.60 years when discounted at $3 \%$ ). The extra cost associated with AN versus AL treatment was $\$ 19,590$ per 1000 cases treated (PP analysis). Therefore, the cost per life year saved was $\$ 19,590 / 3.66=\$ 5352.46$ (or $\$ 12,164.63$ when benefits are discounted at 3\%) for girls and $\$ 19,590 / 3.42=\$ 5728.07$ (or $\$ 12,468.75$ when benefits are discounted at $3 \%$ ) for boys.

\section{Discussion}

This is the first economic analysis of AN for treatment of uncomplicated malaria in an area of intense transmission of multiple Plasmodium species. The present data show that, while a 3-day course of AN had superior overall efficacy in uncomplicated paediatric malaria, the recommended first-line AL remained the more cost-effective treatment. In PNG children with falciparum malaria, AN was equally efficacious but far more costly than AL. In the case of vivax malaria, AN was significantly more efficacious than AL but only slightly more costly in the usual care setting. The most significant contributor to the difference in costs between AN and AL is the fact that AL is a subsidized prequalified first-line therapy provided with financial assistance from a Global Fund Affordable Medicines Facility Grant [13], while AN can only be purchased over-the-counter at pharmacy-determined commercial cost. The availability of donated or subsidized drugs and willingness to pay will, therefore, determine the choice of regimen by the consumer outside the public health system. 
Table 3 Cost-effectiveness analyses by malaria species and type of analysis for a usual care setting

\begin{tabular}{|c|c|c|}
\hline & $A L$ & AN \\
\hline \multicolumn{3}{|l|}{ Plasmodium falciparum } \\
\hline \multicolumn{3}{|l|}{ Per protocol (total $=186)$} \\
\hline Number & 92 & 94 \\
\hline Successes (proportion) & 0.978 & 1.00 \\
\hline Mean cost (US\$)/patient & 7.66 & 17.28 \\
\hline Incremental successes & - & 0.022 \\
\hline Incremental costs (US\$) & - & 9.61 \\
\hline Incremental cost-effectiveness ratio & - & 436.82 \\
\hline \multicolumn{3}{|l|}{ Modified intention to treat $(N=198)$} \\
\hline Number & 100 & 98 \\
\hline \multicolumn{3}{|l|}{ (i) Worst case } \\
\hline Successes (proportion) & 0.900 & 0.959 \\
\hline Mean cost (US\$)/patient & 7.55 & 17.16 \\
\hline Incremental successes & - & 0.059 \\
\hline Incremental costs (US\$) & - & 9.60 \\
\hline \multicolumn{2}{|l|}{ (ii) Best case } & 162.71 \\
\hline Successes (proportion) & 0.980 & 1.000 \\
\hline Mean cost (US\$)/patient & 7.55 & 17.16 \\
\hline Incremental successes & - & 0.020 \\
\hline Incremental costs (US\$) & - & 9.60 \\
\hline Incremental cost-effectiveness ratio & - & 480.00 \\
\hline \multicolumn{3}{|l|}{ Plasmodium vivax } \\
\hline \multicolumn{3}{|l|}{ Per protocol (total = 46) } \\
\hline Number & 20 & 26 \\
\hline Successes (proportion) & 0.300 & 1.000 \\
\hline Mean cost (US\$)/patient & 7.41 & 17.78 \\
\hline Incremental successes & - & 0.700 \\
\hline Incremental costs (US\$) & - & 10.38 \\
\hline Incremental cost-effectiveness ratio & - & 14.83 \\
\hline \multicolumn{3}{|l|}{ Modified intention to treat $(N=47)$} \\
\hline Number & 20 & 27 \\
\hline \multicolumn{3}{|l|}{ (i) Worst case } \\
\hline Successes (proportion) & 0.300 & 0.963 \\
\hline Mean cost (US\$)/patient & 7.41 & 17.52 \\
\hline Incremental successes & - & 0.663 \\
\hline Incremental costs (US\$) & - & 10.11 \\
\hline Incremental cost-effectiveness ratio & - & 15.25 \\
\hline \multicolumn{3}{|l|}{ (ii) Best case } \\
\hline Successes (proportion) & 0.300 & 1.000 \\
\hline Mean cost (US\$)/patient & 7.41 & 17.52 \\
\hline Incremental successes & - & 0.700 \\
\hline Incremental costs (US\$) & - & 10.11 \\
\hline Incremental cost-effectiveness ratio & - & 14.29 \\
\hline
\end{tabular}

Incremental successes and costs are for AN vs. AL

The only formulation of AN currently available is that manufactured by KPC under the brand name $\mathrm{ARCO}^{\circledR}$. It satisfies WHO recommendations for universal combination therapy for uncomplicated malaria but has not yet met the WHO prequalification manufacturing standards [20]. As a result, AN is not eligible for financial subsidy, inclusion on competitive pricing indices, or global distribution by International agencies including UNICEF, the Global Fund and Unitaid [13, 20]. As KPC continues to distribute $\mathrm{ARCO}^{\circledR}$ as a single-dose treatment, a regimen which is inconsistent with WHO recommendations for 3 days of ACT [8], future prequalification appears unlikely. However, given that $\mathrm{ARCO}^{\circledR}$ is marketed in the private sector in a range of countries in Africa, Asia and Oceania, it is important for the costeffectiveness implications of this form of ACT to be assessed.

In PNG, $\mathrm{ARCO}^{\circledR}$ can be purchased as a single-dose therapy across the counter at an average cost of $\$ 1.84$ per tablet, which is significantly more expensive than subsidized AL therapy ( $\$ 0.19$ per tablet) that is usually widely available through government hospitals and clinics $[13,15]$. Even if AN were efficacious as single-dose therapy (as recommended by the manufacturer), its costeffectiveness in comparison to a treatment course of $\mathrm{AL}$ would still be questionable. However, preliminary studies of the safety, efficacy and tolerability of AN in PNG children found unacceptable rates of treatment failure after a single dose $[9,10]$. Dose-ranging studies identified that a minimum of two daily doses was required for adequate Day 28 and Day 42 ACPR, while a 3-day treatment course was considered the most appropriate given WHO recommendations [9].

For falciparum malaria in the present study, AN and AL had similar treatment efficacy (100 and $97.8 \%$ for $\mathrm{AN}$ and AL, respectively) but AL was significantly more cost-effective therapy with a mean cost of US $\$ 7.66$ per patient (PP analysis) compared to US\$17.28 per patient for AN therapy. This finding is consistent with other analyses of AL in a usual care setting. A cost-effectiveness study comparing DHA-PQP and AL for uncomplicated malaria in Tanzanian children completed in 2012 reported a mean cost of US $\$ 8.40$ from the provider's perspective for successful treatment of a clinical case of uncomplicated malaria [21]. Considering currency inflation over a 5-year period, the present findings are also consistent with previous reports of the cost-effectiveness of $\mathrm{AL}$ for treatment of uncomplicated falciparum and vivax malaria in Papua New Guinean children in 2008 (\$6.97 per treatment success) [5]. A potential complication in determining cost-effectiveness analyses across different malaria-endemic regions is the relative lack of data from South-east Asia compared to the African continent where a number of different donation/subsidization schemes ensure availability to all in need [13]. The maximum manufacturer prices for AL for children with 


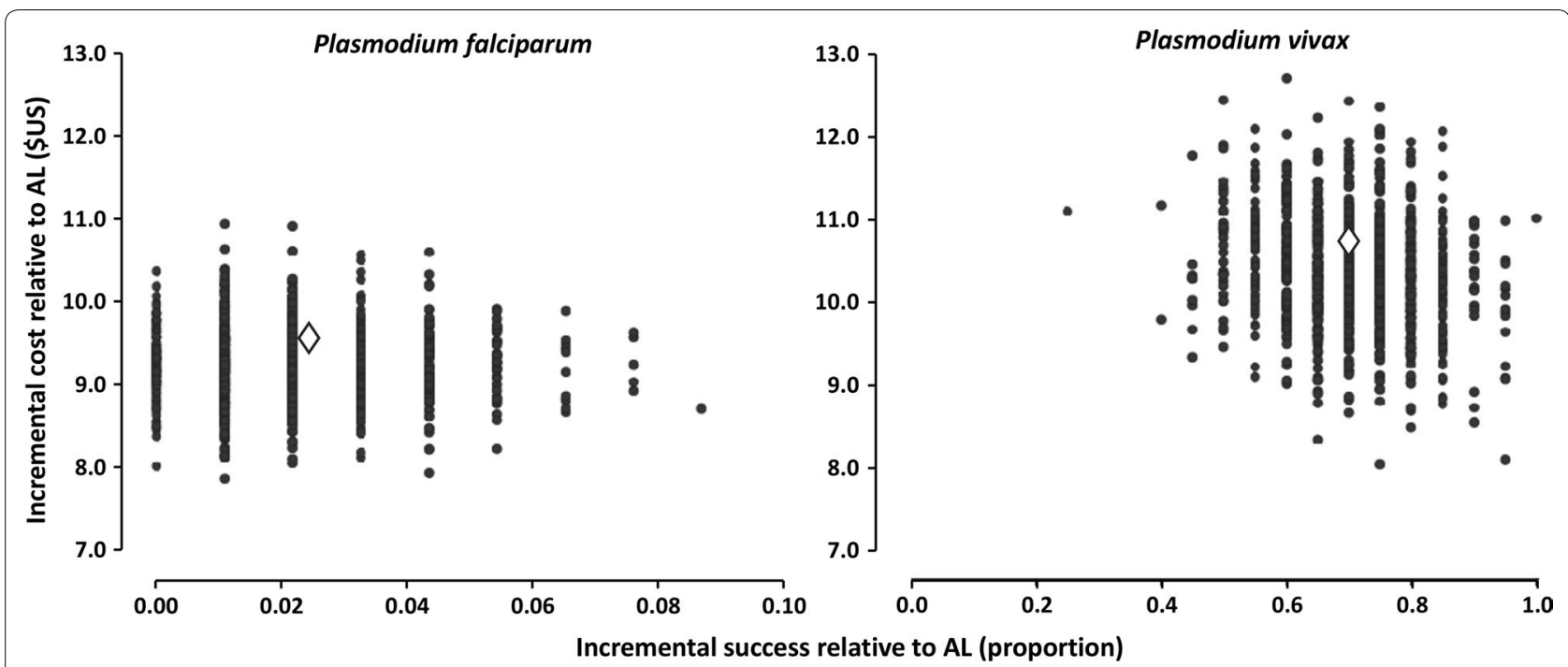

Fig. 2 Scatterplots of estimated joint density of incremental costs and incremental effects (treatment successes) by bootstrap re-sampling of AN relative to AL for children with Plasmodium falciparum malaria (left) and Plasmodium vivax malaria (right) in a usual care setting in Papua New Guinea 2011-2013. The $\diamond$ represents the point estimate for incremental cost and incremental effect (treatment success)

uncomplicated malaria were recently set by the Global Fund at US\$0.43-\$1.22 per treatment course $[12,13]$ but these data are predominantly from African countries and it is not known how many other malaria endemic nations received subsidized anti-malarials under the Affordable Medicines Facility malaria (AMFm) scheme.

A further consideration for countries such as PNG is that, despite a decline in the prevalence of falciparum malaria, the prevalence of $P$. vivax has increased by $13-36 \%$ [22-24] to the point where it has become the predominant Plasmodium species causing infection and illness in young PNG children [25]. Primaquine cannot be given for routine radical cure of $P$. vivax mainly because of the risk of haemolysis due to the relatively high prevalence of glucose-6-phosphatase deficiency in PNG. In this situation, selection of an ACT with a long half-life component is desirable so that there is an extended period of prophylaxis against relapses from hypnozoites during recovery [26]. In the present study, AL was efficacious in only $30 \%$ of children with vivax malaria compared with $100 \%$ after treatment with AN [11]. This rate of AL treatment failure is similar to that reported in other studies conducted in similar geo-epidemiological settings [2729]. It is the most likely reason why the present analysis found AN to be similarly cost-effective to AL in P. vivax infections in contrast to the marked disparity in the case of $P$. falciparum infections. The excess cost per treatment success with AN was $\$ 14.83$ for P. vivax versus $\$ 436.82$ for P. falciparum.

There are population data from PNG detailed enough to provide estimates of the benefit of ACT regimens. In
2009, 1,431,395 suspected malaria cases were treated in PNG with a reported 604 malaria-attributable deaths. This represents $21.3 \%$ of the total PNG population assuming no multiple presentations. Furthermore, a study of febrile patients presenting at five sentinel health facilities across PNG in 2008 reported a malaria slide positivity rate ranging from 2.2 to $74.9 \%$ [30]. The clinical trial data, on which this analysis is based, showed that $79 \%$ of uncomplicated cases were due to $P$. falciparum only, $14 \%$ to $P$. vivax only, and $6 \%$ to mixed $P$. falciparum and $P$. vivax infections. Projecting the trial PP usual care cost-effectiveness analysis to the general PNG population of $7,154,870$ in 2012, and assuming that all suspected cases were confirmed as a worst case scenario, 213,358 and 28,499 more cases of $P$. vivax and $P$. falciparum, respectively, could be treated successfully every year if AN were used instead of AL, costing an extra US $\$ 14,663,345$ per year. However, these estimates may have to be revised as, since the trial was conducted in 2012, two rounds of countrywide distributions of long lasting insecticide treated nets have resulted in a $6.7 \%$ reduction in the overall prevalence of malaria [31, 32]. It was estimated that the cost per life year saved by using AN instead of AL to treat uncomplicated malaria to be $\$ 12,165$ for girls and $\$ 12,469$ for boys (discounted). This may not be cost-effective and affordable given that the gross national income per capita in PNG in 2012 was $\$ 1820$ [33, 34], unless the cost of AN is reduced considerably.

The present study had limitations. The analysis did not consider treatment compliance. All treatments are given 
over 3 days but AL requires two doses per day and the medication should be taken with supplementary fat (usually milk or biscuits), aspects which might reduce compliance. Although all children allocated AL in the trial had a therapeutic plasma lumefantrine concentration on Day 7 , consistent with all six doses having been administered successfully [11], a cost-effectiveness study of ACTs in sub-Saharan Africa estimated compliance with ACTs to be only $30-60 \%$ compared with $85-95 \%$ for sulfadoxinepyrimethamine [35]. As conventionally recommended in cost-effectiveness analyses, estimates were based on the primary endpoints of the intervention trial. In the usual care situation in countries such as PNG, diagnosis and response to treatment are not underpinned by PCR. In addition, microscopy and/or rapid diagnostic tests (RDTs) may not be available or reliable in areas of PNG and in other resource-poor tropical countries. A national cross-sectional survey conducted prior to the implementation of the revised PNG national malaria treatment programme found that that RDTs or functional microscopy was utilized in only $15 \%$ of health facilities [36]. As a result, presumptive treatment based on presenting symptoms is most likely to occur, as shown in the same survey where $96.4 \%$ of fever presentations received anti-malarial treatment including $82 \%$ of patients who tested negative for malaria by either RDT or microscopy [36]. Recent PNG surveys assessing 88 health centres across PNG in 2012 showed a substantial increase in the use of RDTs and microscopy across all health facilities between 2010 and 2012 (16.2\% vs. 68.3\%) [37]. Although an economic analysis of the impact of these changes was beyond the scope of the present study, the data suggest that a progressive reduction in RDT costs in parallel with increased availability may improve diagnostic accuracy in countries such as PNG, and thus reduce inappropriate treatment.

Other potential limitations were that, while uncertainty has been accounted for in the outcomes of the trial via bootstrapping, the estimates of cost per life year are meant to be indicative and so a probabilistic model that would allow quantification of uncertainty around these estimates was not built. The current analysis also did not consider the cost implications of the mixed Plasmodium infections but these were a minor component of the burden of disease (6.5\% of total cases). Post-treatment gametocyte carriage and consequent malaria transmission was also not factored into the analyses. Opportunity costs, such as the time taken off work by parents needing to look after their sick child including transportation to the clinic or hospital, have not been addressed. Treatment that accelerates recovery might allow parents to return to work more quickly. Finally, there are sometimes unpredictable shortages of government supplies of approved drugs such as AL in PNG which means that parents have to fill prescriptions at commercial pharmacies at increased cost.

\section{Conclusions}

AL proved the most cost-effective ACT treatment for both infecting Plasmodium species in PNG but its low efficacy against vivax malaria remains a concern. While, for programmatic reasons, the use of a single first-line therapy that is effective against both $P$. falciparum and $P$. vivax infections is preferred in regions where both species are transmitted, the superiority of AN against vivax malaria $[11,26]$ means that this ACT medicine should be reconsidered if there is a reduction in price and/or it should achieve WHO prequalification status. Since the first intervention trial [5] and related cost-effective analysis [6], DHA-PQP has received approval for WHO prequalification and is, therefore, included on the list of anti-malarial drugs which are subsidized through the Global Fund and AMFm initiative [38]. Given its superior efficacy over $\mathrm{AL}$ for vivax infections and more recent evidence of continued high efficacy in falciparum malaria $[39,40]$, DHA-PQP may need to be changed from second- to first-line treatment in PNG if AL efficacy wanes as has happened in parts of South-east Asia [41]. In the meantime, the present cost-effectiveness data support $\mathrm{AL}$ over $\mathrm{AN}$ for first-line treatment of uncomplicated malaria in PNG and geo-epidemiologically similar areas in Oceania and South-east Asia.

\section{Abbreviations \\ ACPR: adequate parasitological and clinical response; AMF: Affordable Medi- cines Facility malaria; AL: artemether-lumefantrine; ACT: artemisinin-based combination therapy; $\mathrm{AN}$ : artemisinin-naphthoquine; DHA-PQP: dihydroar- temisinin-piperaquine; $\mathrm{Cl}$ : confidence intervals; ETF: early treatment failure; $\mathrm{Hb}$ : haemoglobin; ICER: incremental cost-effectiveness ratio; KPC: Kunming Pharmaceutical Corporation; LCF: late clinical failure; LPF: late parasitological failure; mITT: modified intention to treat; PNG: Papua New Guinea; PP: per protocol; PCR: polymerase chain reaction; RDT: rapid diagnostic test; WHO: World Health Organization.}

\section{Authors' contributions}

TMED designed the intervention trial. BM and ML collected the trial and cost data with logistic and administrative support from LR. WAD and BM analysed the data with technical input from PC. BM and WAD drafted the manuscript, the final version of which was prepared by TMED after input from all authors. All authors read and approved the final manuscript.

\section{Author details}

${ }^{1}$ School of Pharmacy, Curtin University of Technology, Perth, WA, Australia. ${ }^{2}$ School of Medicine and Pharmacology, University of Western Australia, Perth, WA, Australia. ${ }^{3}$ Melbourne School of Population and Global Health, University of Melbourne, Melbourne, Australia. ${ }^{4}$ Papua New Guinea Institute of Medical Research, Madang, Papua New Guinea. ${ }^{5}$ Burnet Institute, Parkville, Melbourne, VIC, Australia. ${ }^{6}$ Division of Population Health and Immunity, Walter and Eliza Hall Institute, Parkville, VIC, Australia.

\section{Acknowledgements}

We gratefully acknowledge the support of patients and their families, staff at the Mugil and Alexishafen Health Centres, and staff in the Vector Borne Disease Unit of the PNG Institute of Medical Research. The main trial was 
funded by a National Health and Medical Research Council of Australia Project Grant (\#634343). BRM was supported by an NHMRC Early Career Fellowship (\#1036951), ML an Australian Award PhD Scholarship, LJR by an NHMRC Early Career Fellowship (\#1016443) and TMED a NHMRC Practitioner Fellowship (\#572561).

\section{Competing interests}

The authors declare that they have no competing interests.

\section{Publisher's Note}

Springer Nature remains neutral with regard to jurisdictional claims in published maps and institutional affiliations.

Received: 21 July 2017 Accepted: 21 October 2017

Published online: 30 October 2017

\section{References}

1. WHO. Morld Malaria Report. Geneva: World Health Organization; 2016.

2. Murray CJL, Barber RM, Foreman KJ, Ozgoren AA, Abd-Allah F, Abera SF, et al. Global, regional, and national disability-adjusted life years (DALYs) for 306 diseases and injuries and healthy life expectancy (HALE) for 188 countries, 1990-2013: quantifying the epidemiological transition. Lancet. 2015;386:2145-91.

3. Gething PW, Elyazar IR, Moyes CL, Smith DL, Battle KE, Guerra CA, et al. A long neglected world malaria map: Plasmodium vivax endemicity in 2010. PLoS Negl Trop Dis. 2012;6:e1814.

4. Papua New Guinea National Department of Health. National malaria treatment protocol. Port Moresby: National Department of Health; 2009.

5. Karunajeewa HA, Mueller I, Senn M, Lin E, Law I, Gomorrai PS, et al. A trial of combination antimalarial therapies in children from Papua New Guinea. N Engl J Med. 2008;359:2545-57.

6. Davis WA, Clarke PM, Siba PM, Karunajeewa HA, Davy C, Mueller I, et al. Cost-effectiveness of artemisinin combination therapy for uncomplicated malaria in children: data from Papua New Guinea. Bull World Health Organ. 2011:89:211-20.

7. Manning L, Laman M, Law I, Bona C, Aipit S, Teine D, et al. Features and prognosis of severe malaria caused by Plasmodium falciparum, Plasmodium vivax and mixed Plasmodium species in Papua New Guinean children. PLOS ONE. 2011;6:e29203.

8. WHO. Guidelines for the treatment of malaria. 2nd ed. Geneva: World Health Organization; 2010.

9. Benjamin J, Moore B, Lee ST, Senn M, Griffin S, Lautu D, et al. Artemisininnaphthoquine combination therapy for uncomplicated pediatric malaria: a tolerability, safety, and preliminary efficacy study. Antimicrob Agents Chemother. 2012;56:2465-71.

10. Batty KT, Salman S, Moore BR, Benjamin J, Lee ST, Page-Sharp M, et al. Artemisinin-naphthoquine combination therapy for uncomplicated pediatric malaria: a pharmacokinetic study. Antimicrob Agents Chemother. 2012;56:2472-84.

11. Laman M, Moore BR, Benjamin JM, Yadi G, Bona C, Warrel J, et al. Artemisinin-naphthoquine versus artemether-lumefantrine for uncomplicated malaria in Papua New Guinean children: an open-label randomized trial. PLoS Med. 2014;11:e1001773.

12. Pfeil J, Borrmann S, Tozan Y. Dihydroartemisinin-piperaquine vs. artemether-lumefantrine for first-line treatment of uncomplicated malaria in African children: a cost-effectiveness analysis. PLoS ONE. 2014;9:e95681.

13. The Global Fund. Antimalarial Medicines-ACT Reference Pricing (April 2016). http://www.theglobalfund.org/en/sourcing/acts/. Accessed June 2017.

14. World Health Organization. World Malaria Report. Geneva: World Health Organization; 2010.

15. National Department of Health, World Health Organization. Medical and dental catalogue. 10th ed. Papua New Guinea: Government of Papua New Guinea; 2012.

16. UKForex. Currency Tools. http://www.ukforex.co.uk/forex-tools/histororical-rate-tools/historical-exchange-rates. Accessed June 2017.
17. Campbell MK, Torgerson DJ. Bootstrapping: estimating confidence intervals for cost-effectiveness ratios. QJM. 1999;92:177-82.

18. WHO. Global Health Observatory data repository; Papua New Guinea. Geneva: World Health Organization. http://apps.who.int/gho/data/node. country.country-PNG?lang=en. Accessed June 2017.

19. WHO. Global Health Observatory data repository: Life tables by country-Papua New Guinea. Geneva: World Health Organization. http://apps. who.int/gho/data/view.main.61260?lang=en. Accessed June 2017.

20. WHO. Prequalification of medicine by WHO. Geneva: World Health Organization. http://www.who.int/mediacentre/factsheets/fs278/en/. Accessed June 2017.

21. Mori AT, Ngalesoni F, Norheim OF, Robberstad B. Cost-effectiveness of dihydroartemisinin-piperaquine compared with artemether-lumefantrine for treating uncomplicated malaria in children at a district hospital in Tanzania. Malar J. 2014;13:363.

22. Arnott A, Barnadas C, Senn N, Siba P, Mueller I, Reeder JC, et al. High genetic diversity of Plasmodium vivax on the north coast of Papua New Guinea. Am J Trop Med Hyg. 2013;89:188-94.

23. Schultz L, Wapling J, Mueller I, Ntsuke PO, Senn N, Nale J, et al. Multilocus haplotypes reveal variable levels of diversity and population structure of Plasmodium falciparum in Papua New Guinea, a region of intense perennial transmission. Malar J. 2010;9:336.

24. Mueller I, Widmer S, Michel D, Maraga S, McNamara DT, Kiniboro B, et al. High sensitivity detection of Plasmodium species reveals positive correlations between infections of different species, shifts in age distribution and reduced local variation in Papua New Guinea. Malar J. 2009:8:41.

25. Robinson LJ, Wampfler R, Betuela I, Karl S, White MT, Li Wai Suen CSN, et al. Strategies for understanding and reducing the Plasmodium vivax and Plasmodium ovale hypnozoite reservoir in Papua New Guinean Children: a randomised placebo-controlled trial and mathematical model. PLoS Med. 2015;12:e1001891.

26. Bassat $\mathrm{Q}$. The use of artemether-lumefantrine for the treatment of uncomplicated Plasmodium vivax malaria. PLoS Negl Trop Dis. 2011;5:e1325

27. Senn N, Rarau P, Manong D, Salib M, Siba P, Reeder JC, et al. Effectiveness of artemether/lumefantrine for the treatment of uncomplicated Plasmodium vivax and P. falciparum malaria in young children in Papua New Guinea. Clin Infect Dis. 2013;56:1413-20.

28. Yohannes AM, Teklehaimanot A, Bergqvist $Y$, Ringwald P. Confirmed vivax resistance to chloroquine and effectiveness of artemether-lumefantrine for the treatment of vivax malaria in Ethiopia. Am J Trop Med Hyg. 2011:84:137-40.

29. Ratcliff A, Siswantoro H, Kenangalem E, Maristela R, Wuwung RM, Laihad F, et al. Two fixed-dose artemisinin combinations for drug-resistant falciparum and vivax malaria in Papua, Indonesia: an open-label randomised comparison. Lancet. 2007;369:757-65.

30. Hetzel MW, Paul S, Benjamin L, Makita L, Mueller I, Siba PM. The proportion of fevers attributable to malaria varies significantly between sites in Papua New Guinea. P N G Med J. 2014;57:39-51.

31. WHO. World Malaria Report. Geneva: World Health Organization; 2012.

32. Killeen GF, McKenzie FE, Foy BD, Schieffelin C, Billingsley PF, Beier JC. The potential impact of integrated malaria transmission control on entomologic inoculation rate in highly endemic areas. Am J Trop Med Hyg. 2000;62:545-51.

33. WHO. Data: Papua New Guinea. Geneva: World Health Organization. http://data.worldbank.org/country/papua-new-guinea. Accessed June 2017.

34. Shillcutt SD, Walker DG, Goodman CA, Mills AJ. Cost effectiveness in low- and middle-income countries: a review of the debates surrounding decision rules. Pharmacoeconomics. 2009;27:903-17.

35. Coleman PG, Morel C, Shillcutt S, Goodman C, Mills AJ. A threshold analysis of the cost-effectiveness of artemisinin-based combination therapies in sub-saharan Africa. Am J Trop Med Hyg. 2004;71:196-204.

36. Kurumop SF, Pulford J, Mueller I, Siba PM, Hetzel MW. Diagnostic capacity and antimalarial availability in Papua New Guinea before the introduction of a revised national malaria treatment protocol. P N G Med J. 2014;57:59-67.

37. Pulford J, Kurumop SF, Ura Y, Siba PM, Mueller I, Hetzel MW. Malaria case management in Papua New Guinea following the introduction of a revised treatment protocol. Malar J. 2013;12:433. 
38. Wells TNC, van Huijsduijnen RH, Van Voorhis WC. Malaria medicines: a glass half full? Nat Rev Drug Discov. 2015;14:424-42.

39. Benjamin JM, Moore BR, Salman S, Page-Sharp M, Tawat S, Yadi G, et al. Population pharmacokinetics, tolerability, and safety of dihydroartemisinin-piperaquine and sulfadoxine-pyrimethamine-piperaquine in pregnant and nonpregnant Papua New Guinean women. Antimicrob Agents Chemother. 2015;59:4260-71.
40. Moore BR, Benjamin JM, Salman S, Griffin S, Ginny E, Page-Sharp M, et al. Effect of coadministered fat on the tolerability, safety, and pharmacokinetic properties of dihydroartemisinin-piperaquine in Papua New Guinean children with uncomplicated malaria. Antimicrob Agents Chemother. 2014;58:5784-94.

41. Denis MB, Tsuyuoka R, Lim P, Lindegardh N, Yi P, Top SN, et al. Efficacy of artemether-lumefantrine for the treatment of uncomplicated falciparum malaria in northwest Cambodia. Trop Med Int Health. 2006;11:1800-7.

\section{Submit your next manuscript to BioMed Central and we will help you at every step:}

- We accept pre-submission inquiries

- Our selector tool helps you to find the most relevant journal

- We provide round the clock customer support

- Convenient online submission

- Thorough peer review

- Inclusion in PubMed and all major indexing services

- Maximum visibility for your research

Submit your manuscript at www.biomedcentral.com/submit
() Biomed Central 